\title{
Standardized Patient Encounters and Individual Case-Based Simulations Improve Students' Confidence and Promote Reflection: A Preliminary Study
}

Stacy Walker, PhD, ATC*; Thomas Weidner, PhD, ATC*; Kirk J. Armstrong, EdD, LAT, ATC $†$ *School of Physical Education, Sport and Exercise Science, Ball State University, Muncie, IN; $†$ School of Health and Human Performance, Georgia College \& State University, Milledgeville

Context: Simulations and standardized patient (SP) encounters are used to provide clinical experiences for students. In athletic training, no research has examined the perceived educational benefits of these interventions.

Objective: To explore athletic training students' perceptions regarding small group SP encounters and individual casebased simulations (CBSs).

Design: Grounded theory.

Setting: One midwestern university.

Patients or Other Participants: Nine athletic training students ( 3 males, 6 females; $20 \pm 0.833$ years old) who enrolled in a lower extremity orthopaedic evaluation course during their first or second semester in an athletic training program.

Main Outcome Measure(s): Semistructured interviews were recorded, transcribed verbatim, and analyzed using open coding and axial and selective coding (ie, to develop themes/threads). To ensure trustworthiness, we used member checks and peer debriefing.

Results: For both the small group SP counter and individual CBS, 2 themes emerged: (1) reflection-on-specific action and (2) increased confidence. Participants reflected on both experiences when providing patient care. Participants felt that both encounters could improve their confidence with regard to future clinical evaluations. Specific to the small group SP encounters, peer-assisted learning emerged as a theme. Participants felt they learned from and with each other when performing the evaluation together. Specific to the individual CBS, organization of thoughts emerged as a theme. During the individual CBSs, participants felt they developed, controlled, and organized their thoughts during the evaluation.

Conclusions: Both teaching encounters can be integrated into the curriculum of an athletic training program. Each strategy provides students with a nonthreatening educational experience with no harm to patients. In addition, each encounter can correspond to content taught in the classroom or laboratory. The long-term benefits of small group SP encounters and CBSs are unclear, and more research is needed.

Key Words: Schema, clinical education, peer teaching

Dr Walker is currently an Associate Professor of Athletic Training at Ball State University. Please address all correspondence to Stacy Walker, PhD, ATC, School of Physical Education, Sport and Exercise Science, Ball State University, 2000 West University Avenue, Muncie, IN 47306. sewalker@bsu.edu.

Full Citation:

Walker S, Weidner T, Armstrong KJ. Standardized patient encounters and individual case-based simulations improve students' confidence and promote reflection: a preliminary study. Athl Train Educ J. 2015;10(2):130-137. 


\title{
Standardized Patient Encounters and Individual Case-Based Simulations Improve Students' Confidence and Promote Reflection: A Preliminary Study
}

\author{
Stacy Walker, PhD, ATC; Thomas Weidner, PhD, ATC; Kirk J. Armstrong, EdD, LAT, ATC
}

\section{INTRODUCTION}

Athletic training students need a variety of clinical experiences to help them grow into their roles as healthcare professionals. Unfortunately, we are unable to guarantee that the best exposure to these experiences will occur for each student during his clinical education. Simulations and standardized patient (SP) experiences are teaching methods that help students gain confidence $^{1}$ and can ensure that students are exposed to specific clinical situations, such as emergencies (eg, fracture, joint dislocation, myocardial infarction) and complex injuries (eg, concussions). Simulations and SP encounters also provide students the opportunity to develop and practice clinical decision-making skills in a safe manner at a time that coincides with their progression through the professional program.

A recent survey of athletic training program directors found that $76 \%$ have used SP encounters and simulations to evaluate athletic training clinical proficiencies and that they did so more than $50 \%$ of the time. ${ }^{2}$ A SP encounter includes an individual who has been trained to portray the signs and symptoms of a particular condition, injury, or illness (eg, shoulder pain, acute cervical spine injury) in a consistent fashion. ${ }^{1}$ In contrast, a simulation is defined as a scenario or clinical situation in which an untrained mock patient, who is typically a peer student or preceptor, has had no training in portraying an injury or condition in a standardized and consistent fashion.

Though real-time patient encounters are actually preferred to simulations, there are often insufficient opportunities for students to perform these real-time evaluations. Barriers to these opportunities can include an inadequate volume of injuries and/or patient care, which is more of a priority over the student's clinical education. ${ }^{2}$ Preceptors report that simulations are used $79.7 \%$ of the time to evaluate a student's clinical proficiencies. ${ }^{3}$ The most reported barrier to the preferred timing of clinical encounters was that the timing of the injury did not often coincide with the timetable of the needed clinical proficiency evaluation. ${ }^{3}$ Standardized patient encounters are being widely used in other healthcare professional programs to teach and evaluate clinical and communication skills. These include programs in nursing 4,5 (eg, care of a paralyzed patient, pelvic exams), physical therapy $^{6-8}$ (eg, gait training, ethical decisions), and medicine ${ }^{9}$ (eg, delivering bad news, physical examination).

Because students best develop clinical competence if given the opportunity to use the skills they learn in a timely and realistic way, ${ }^{3}$ it is worth investigating how these SP encounters and simulations affect learning among athletic training students. Both SPs and simulations appear to be used to evaluate and teach athletic training students, but how these experiences are perceived by athletic training students is unknown and has not been researched. The purpose of this investigation was to explore athletic training students' perceptions regarding small group SP encounters and simulations.

\section{METHODS}

\section{Participants}

Nine athletic training students (3 males, 6 females; $20 \pm$ 0.833 years old $)$ in their first $(\mathrm{n}=5)$ or second semester $(\mathrm{n}=$ 4) in the professional phase of an athletic training program at a midwestern university participated in this investigation. One participant's data was not analyzed as the result of a recording device error. All participants were sophomores or juniors, enrolled in a lower extremity orthopaedic evaluation course, and received the same classroom and laboratory instruction.

\section{Procedures}

Before initiating this investigation, we obtained approval from the institutional review board. Using a counterbalance design to control for level of student (ie, semester in the program), we randomly assigned all participants to 1 of 2 groups (group A or B). The week after classroom/laboratory instruction on ankle evaluation (approximately 6 weeks into the semester), participants in group A completed a small group SP encounter. Participants in Group B completed an individual case-based simulation (CBS) during the same week. The week after classroom/laboratory instruction on knee evaluation (approximately 11 weeks into the semester), participants in group B then completed a small group SP encounter. Participants in group A completed an individual CBS during the same week. The mock ankle and knee injuries for both the small group SP encounter and individual CBS were the same for all participants. Four weeks later (approximately 15 weeks into the semester), we interviewed each participant. The Figure displays a timeline of the procedures.

Figure. Timeline of procedures. Abbreviation: SP, standardized patient.

\begin{tabular}{|c|c|}
\hline Group A & Group B \\
\hline $\begin{array}{c}\text { Ankle Evaluation } \\
\text { Classroom instruction }\end{array}$ & $\begin{array}{c}\text { Ankle Evaluation } \\
\text { Classroom instruction }\end{array}$ \\
\hline $\begin{array}{c}\text { Small Group SP } \\
\text { Encounter }\end{array}$ & Case-Based Simulation \\
\hline $\begin{array}{c}\text { Knee Evaluation } \\
\text { Classroom Instruction }\end{array}$ & $\begin{array}{c}\text { Knee Evaluation } \\
\text { Classroom Instruction }\end{array}$ \\
\hline $\begin{array}{c}\text { Small Group SP } \\
\text { Encounter }\end{array}$ \\
\hline Individual Interviews
\end{tabular}




\section{Interventions}

Small Group Standardized Patient Encounter. The small group SP encounters consisted of 3 or 4 randomly selected participants placed into a group. The participants in a group evaluated as a team a trained SP (theater major) with the instructor (primary investigator) and a preceptor/clinician present. The participants were instructed to interact with the SP as they would with a normal patient/athlete, to conduct an orthopaedic evaluation, and to form a treatment plan together as a group.

We used the time-in, time-out instructional procedure, which was explained to the participants. ${ }^{2,10}$ This procedure is used to provide students with immediate feedback, which can be useful during the SP encounter. The instructor and preceptor/ clinician did not intervene or speak during the encounter unless a time-out was called. When the participants became overwhelmed and/or were not sure how to proceed with the evaluation, and/or the instructor or preceptor/clinician wanted to intervene, a time-out was called. Time-outs included when participants used confrontational phrasing of questions (eg, "Why didn't you seek treatment sooner?") and promises to the SP regarding the patient's return to play that could not be guaranteed. During this time-out the participants, instructor, clinician/preceptor, and SP discussed the participants' approach to the evaluation and any issues or confusion on the part of the participants (eg, unsure how to discuss with the patient the need to talk to her parents about the injury). These conversations would not normally happen in the presence of a patient. Feedback regarding issues such as speaking tone, body language, phrasing and depth of the history questions, and proper physical examination techniques (eg, compare bilaterally) were also discussed. When the discussion and feedback was over, the instructor directed participants to continue their interaction with the SP (ie, timein).

In addition to the mentioned examples of feedback, we found most participants needed to be reminded during a time-out that they were evaluating a person as a result of the SP commenting on how she felt the participants didn't truly care about her but instead cared about diagnosing the injury. Even with initial instructions to evaluate the patient, some participants were focused on diagnosing the condition and failed to engage in small talk or any other discussions with the SP (other than questions relating to the diagnosis).

Each small group SP encounter lasted approximately 45 to 60 minutes and took place in the Athletic Training Research and Education Laboratory. A debrief session occurred after the small SP group encounter. This debrief session consisted of participants discussing their feelings, successes, and challenges during the encounter as well as obtaining feedback from the instructor, clinician/preceptor, and SP. During the encounter and debrief session, participants were given verbal feedback on their performance (eg, clinical skills, hand placement, tone and phrasing of questions, nonverbal communication).

Standardized Patient Case Development and SP Training. The SP cases were developed using a template adapted from the Clinical Performance Center at the University of Illinois at Chicago Department of Medical Education (used with permission). ${ }^{1}$ The foot/ankle case
Table 1. Cases Used for Small Standardized Patient Group Encounter and Individual Case-Based Simulation

This case involves a 17-year-old male/female soccer player who complains of knee pan. The location of this encounter is the athletic training room during halftime of a soccer match. The student is to evaluate the condition and provide verbal feedback.

This case involves a 17-year-old male/female high school basketball player who is told by the coach to visit the athletic trainer because of limping. The location of this encounter is the athletic training room. The student is to evaluate the condition and provide verbal feedback.

involved a 17-year-old female basketball player complaining of foot pain who had a metatarsal stress fracture. The knee case involved a 17-year-old soccer player complaining of knee pain who had a first-degree medial collateral ligament sprain. Both cases were developed by the primary investigator along with a preceptor/clinician using information from the literature and actual patient encounters in clinical practice. Each case consisted of the following elements: summary of the case, student instructions, and SP training materials. A general summary of both cases is provided in Table 1 . The cases were then evaluated for accuracy of content and/or fidelity by an educator with SP research experience, an athletic training educator, and a clinician. As in our previous research, ${ }^{2}$ theater majors were recruited to serve as SPs. Each of the 2 SPs used in this study underwent 5 hours of training with the primary investigator. This training consisted of an orientation to the study and the specific case (ankle/foot or knee) and instructions on how to provide adequate feedback to the participants during the orthopaedic evaluation. Review sessions with the SPs occurred 30 minutes before each actual small group encounter with the students.

Case-Based Simulation. For the purposes of this article, we refer to simulations as "case-based," as they were preplanned around specific criteria. The brief description in the written template for the SP small group encounter was used to prepare the mock patient in the individual CBS and is provided in Table 1. Participants in the individual CBS group evaluated a mock patient (senior athletic training student) while only the instructor (primary investigator) was present. The mock patient/athlete (same individual used throughout the study) did not undergo any training or practice but was privately briefed on the case for 3 to 5 minutes by the instructor using written information about the case. This information was also provided to the participant. This briefing focused on the chief complaint, location and level of pain, any mechanism of injury or pertinent history directly relating to the injury, and any key signs and symptoms that should be reported to the participant during the evaluation. The participants were told they needed to perform an orthopaedic evaluation and form a treatment plan for the patient. During the evaluation, the participant was given no assistance from the mock patient or the instructor. The instructor only intervened if the participant asked the mock patient a question that the mock patient was unable to answer (eg, "Do you have swelling?"). Each individual CBS lasted approximately 20 to 30 minutes and took place in the Athletic Training Research and Education Laboratory. When completed, each participant received verbal feedback (eg, clinical skills, hand placement, tone and phrasing of questions, 


\section{Table 2. Semistructured Interview Questions}

1. Provide an example as to how the small group SP encounter has influenced your evaluation of a patient in the past 4 weeks. What did you do or not do because of that experience?

2. Provide an example as to how the individual CBS has influenced your evaluation of a patient in the past 4 weeks. What did you do or not do because of that experience?

3. Which experience did you prefer, the small group SP encounter or the individual CBS?

4. How did the small group SP encounter change the way you evaluated a patient during your clinical experiences?

5. How did the individual CBS change the way you evaluated a patient during your clinical experiences?

6. How did the small group SP encounter affect your clinical decision-making confidence during your clinical experiences?

7. How did the individual CBS affect your clinical decisionmaking confidence during your clinical experiences?

Abbreviations: SP, standardized patient; CBS, case-based simulation.

nonverbal communication) regarding her performance from both the instructor and the mock patient.

\section{Data Collection and Analysis}

We used an exploratory qualitative method of inquiry to identify athletic training students' perceptions regarding small group SP encounters and individual CBSs. Semistructured audiotaped interviews lasting approximately 30 minutes with each participant took place in a conference room. One participant's data were not analyzed as the result of a recording device error. We developed the semistructured interview questions (Table 2) using previous experience with SPs and the purpose of our study. We transcribed interviews verbatim, and we analyzed data using open coding and axial and selective coding (ie, to develop themes/threads). ${ }^{11}$ Data saturation occurred at interview 5, but we continued the interviews to obtain all participants' perspectives. We first assessed general perceptions associated with participating in CBS and small group SP encounters, and we analyzed the comments from the participants together. Specific comments associated with the individual CBSs and the small group SP encounters were then analyzed separately. To ensure trustworthiness, we used member checks ( 3 participants reviewed the transcript for accuracy) and peer debriefing. We performed peer debriefing using a qualitative research expert in athletic training. This individual reviewed the transcripts as well as our themes for relevance, consistency, and logic.

\section{RESULTS}

After analyzing all of the data together (data related to individual CBS and small group SP encounters) 2 overarching themes emerged: (1) reflection-on-specific action and (2) increased confidence. In addition, subthemes also emerged regarding the SP small group encounter and individual CBS encounters. We used pseudonyms to protect the participants' identities.

\section{Reflection-on-Specific Action}

Reflection-on-specific action involves reflecting on past experiences and thinking about future actions. ${ }^{12}$ In the case of the participants, this entailed considering future actions with patients. One participant, Sayid, described how he reflected back on his SP group encounter:

Something that the preceptor said during my group experience was the way I was forming my sentences in communication with my athlete. Possibly giving them the wrong perception of something, and ever since then, I do think about what I am going to say before I say it and how they are going to hear it and how they are going to perceive it, so that way I'm not giving them a false sense of hope.

As described in this quote, Juliette described how she reflected back to the small SP group encounter and how her reflection changed her future patient care:

During the small SP group encounter, I said that I would probably have them kick a soccer ball to determine their function. I had really never thought of doing that with an athlete before. I don't know why I never thought about that before, but I thought of it when I was doing the experience. Recently I was interacting with a patient who was not in season but her shoulder hurt and she wanted to participate. She wanted to know if she could play and I had her actually take the stick we had in there and swing it [field hockey stick] and tell me how she felt to determine her function.

Another participant, Benjamin, when referring to the individual CBS, discussed how he could be more thorough on future evaluations:

Our model had the MCL [medial collateral ligament] problem, and that's all I tested for. I didn't check for [the] ACL [anterior cruciate ligament] or things like that. And then I just thought afterwards, oh, it was a good idea to rule things out and check for ACL problems and all that because just because the pain is right there doesn't mean something else couldn't be [a] problem.

Participants reflected back to both their experience of the individual CBS as well as the small SP group encounter during their clinical education. Claire also discussed how she now reflects back on both of the experiences and thinks, "If I were doing that [evaluation], again, how would I go about it?"

\section{Increased Confidence}

Participants perceived that both experiences increased their confidence to perform future evaluations. Sayid stated the following:

Before we did these experiences, especially individual [CBS], I was always kind of timid and would shy away from an evaluation because I didn't feel comfortable with my skills. After I did the individual [CBS], I ran into the opportunity to help my preceptor with a shoulder evaluation. Normally I would have gotten the preceptor and immediately have her help me. With the boost of confidence I got from the individual [CBS] experience, I jumped right in, started on the evaluation, got to a point where I thought I would need a little help, and then went to ask my preceptor for help.

Richard commented on how the SP small group encounter affected their confidence: "The part where we had trouble on how to talk to the patient about seeing a physician and talking 
to their parents as to possible return to play, that helped me a lot, because I had never really thought about that." One participant, Rose, commented on how both experiences increased her confidence level with regard to future evaluations: "I felt a little more comfortable because I felt a little more accomplished. Knowing that I could do it and especially being first semester, just knowing that you're able to complete it and knowing what you're doing makes you feel better." Similarly, Benjamin commented, "I felt a little more prepared. I kind of liked the group one [small SP group], how it made me feel good we got it [the diagnosis] right. I felt like I could actually do this. It gave me a little more confidence." Helen stated, "I felt more confident, I guess, so that I know that I can do it, especially when I did my individual one. I felt like I could do it myself. It gave me a little bit more confidence, to say that "oh, I can really do this."

\section{Standardized Patient Small Group Encounter}

One theme emerged from SP small group encounters. The theme "peer learning" refers to how the participants learned with and from each other. One participant, Juliette, commented about this learning, "not that I rely on people, but I just feel like I don't stress out as much and its easier for me to bounce ideas off of people, rather than to me personally freak out and go 'am I right?"' Richard also felt he learned from and evaluated the patient together with his peers. He stated, "we rocked that thing. I mean, because we all helped equally and one of us would say something ... I mean, with the history, we really tried to figure out what happened. I liked how we worked together." Libby commented on how participants in her group shared ideas regarding the evaluation "by getting other opinions, in the group one, one of us was thinking one thing, and another person was thinking another, so we compared what we thought." Another participant, Charlotte, also valued being able to discuss the group's decisions and to learn from peers: "I liked it better because you have other people there to bounce ideas off of, I felt a little more confident in the group." Helen commented:

I liked my group encounter more, it gave us more ideas to bounce off each other so that we could come together as a conclusion. If one person didn't think about something, someone else was there to think about it. I think it just worked out really well.

Charlotte felt she learned from her peers during the small SP group evaluation: "it gave you an idea of how other people did their evaluations compared to how you do yours. It taught me different ways to do it."

\section{Case-Based Simulation}

Organization of thoughts emerged as a theme from the CBSs. This theme describes how the participants felt the CBS helped them begin to develop, control, and organize their thoughts during the evaluation. The participants felt that during the CBSs they could control and organize their thoughts. For example, Rose commented, "It was more like understanding. You learn how to do it in class, but then when you actually have to do it, it's different." Rose also spoke to how the CBS helped her "control the environment and you were able to control your thoughts." Benjamin commented on how he thought back to his CBS encounter: "I just did it [the evaluation] through my head. I just played it [the evaluation] through my head, okay, you do this first, it wasn't on an actual person, but like a thought process." Many participants commented on how the experience engaged them in an evaluation differently because they could not "bounce ideas" off each other. Participants had to refer to their prior knowledge and organize their thoughts because they were performing the evaluation without their peers. Juliette stated that the CBS helped her: "it [CBS] made me thinking about things ... rather than "oh my gosh, what do I do first." Claire commented, "I liked the one-on-one better, in a way, because it was just myself, and I was still hesitant but I knew what you had to do, and you couldn't look at the other people and ask them what they are thinking." Richard echoed this same sentiment: "because it was all me, with the individual, it's all yourself."

\section{DISCUSSION}

\section{Confidence}

We explored athletic training students' perceptions regarding the benefits of small group SP encounters and individual CBSs. We found that both experiences increased their confidence and engaged participants to think about their approach to patient care. Previous research ${ }^{1}$ with athletic training students found that one-on-one encounters with SPs increased students' confidence on future evaluations. Our research highlights that a SP small group encounter and an individual CBS are additional learning experiences that also increase students' confidence. Participants commented that they felt more "accomplished" and/or "experienced" after these encounters. Knight ${ }^{13}$ and Scriber and Trowbridge ${ }^{14}$ speculated that athletic training students struggle to obtain an adequate level of confidence during clinical education. A variety of reasons may exist as to why students struggle to obtain adequate confidence, one of which may be lack of exposure to patients during clinical experiences. Our study has shown that increasing students' exposure to patient care situations left them feeling more accomplished. Both CBS and SP encounters can be used to supplement clinical education and to provide students with additional patient encounters. After each experience in this study, the participants felt more accomplished because they had performed a realistic evaluation and were provided with immediate feedback. As stated above, one participant (Sayid) reported that after engaging in these experiences, specifically the individual CBS, he initiated an evaluation on a patient. In the past he may have been hesitant to initiate an evaluation.

\section{Reflection-on-Specific Action}

Participants felt both the SP small group encounter and the individual CBS provided them with an experience to reflect and consider future approaches and actions regarding the evaluation of a patient. Participants reported using these experiences to plan and perform future patient care. Reflection of past experiences has been found to inform future clinical decision making and is a necessary component of developing reasoning skills. ${ }^{12}$ Novice (those with less than 1 year of experience) and expert (those with 8 years of experience) physical therapists were examined to determine what type and extent of reflection inform their clinical decision making and how reflection influences their decision making. ${ }^{12}$ The novice and expert physical therapists were observed during evaluation and treatment of a patient and 
were videotaped during various patient encounters. They were then interviewed, and while watching videotaped segments of themselves providing patient evaluations and treatments, they thought aloud to explain their decision-making process. ${ }^{12}$ Both novice and expert physical therapists engaged in reflected-on-specific action more than the other types of reflection, such as reflecting-on-professional experience and reflection-in-action. ${ }^{12}$ Reflection-on-specific action occurs after an activity during which one is self-assessing his own performance, and the athletic training students engaged in this type of reflection during the individual CBS and small SP group encounter. Novice and expert physical therapists reflected back on both experiences to plan and decide on future evaluations. ${ }^{12}$

In another study, ${ }^{8}$ third-year medical students individually evaluated SPs during a 6-station clinical skills examination. At each station, the student interacted with an SP who portrayed a case representing a spectrum of third-year clerkship experiences (eg, depression, acute abdominal pain, headache). Typically, when students participate in a clinical skills examination (ranging from 6 to 15 stations), students must complete each station and are not allowed to revisit a station but instead must continue to the next station. As part of this study to simulate real medical practice, the medical students were allowed to revisit their last 3 stations/SPs to ask any additional questions after thinking about the patient encounter. The students were evaluated by the SP via a checklist. This checklist evaluated their medical skills and patient satisfaction. Both skills and satisfaction were statistically significantly higher on the stations that students could revisit. The opportunity to revisit the stations was perceived by the medical students to enhance their decision making, patient education/counseling skills, and confidence. ${ }^{8}$

Reflection is at the heart of education, ${ }^{15}$ and as athletic training educators, we should teach and encourage students to reflect on past experiences. ${ }^{16,17}$ While we know students engage in reflection, more insight is needed to further identify how students use reflection as well as how clinicians do the same. Students are prepared for the knowledge and skills to provide patient care, but as they progress through their education, they must also practice, enhance, and habitually use their reflection skills. ${ }^{17}$ Reflection could develop a student's clinical decision making, and it is recommended that academics and preceptors develop and structure learning activities to promote and develop clinical decision making and reflection. ${ }^{18}$

\section{Peer-Assisted Learning}

Specific to the small group SP experiences, peer-assisted learning emerged as a theme. Peer-assisted learning is the act or process of gaining knowledge, understanding, or skill in athletic training tasks among students who are either at different or the same academic or experience level through instruction and experience. ${ }^{19}$ Participants learned from one another by sharing their thoughts and, together, engaging in the decision-making process. Participants were able to compare their evaluation process to those of others and also to learn different approaches to their evaluation process. The participants in this investigation engaged in intentional team/ group activities, but peer-assisted learning does occur naturally during clinical education. ${ }^{19}$
The small group size offers opportunities for interactive demonstration and student participation..$^{20}$ In our study, peerassisted learning occurred with students in groups. In athletic training only, one-on-one encounters have been researched. Weidner and Popp ${ }^{21}$ found that after engaging in peer learning students felt less anxious performing psychomotor skills and more self-confident when practicing skills with a peer tutor versus an instructor. Students also felt that engaging with peer tutors increased their collaboration with other students. A more recent study 22 investigated pretest and posttest scores on therapeutic modality (eliciting a muscle contraction and pain control) and orthopaedic evaluation (foot and toes, ankle and lower leg, elbow, thoracic) psychomotor skills. It was found that peer interaction can increase student skill performance scores for the peer-tutors.

In a study ${ }^{23}$ with second-year and third-year nursing students, focus groups were used to explore their experience in a clinical practicum. Students reported that peer learning reinforced their knowledge, enhanced their confidence, and allowed them to share experiences. In another study ${ }^{20}$ first-year medical students, in different groups of 10 , interacted with a highfidelity simulator to enhance their understanding of cardiovascular physiology. Confidence in their understanding of the cardiovascular physiology concepts significantly improved as a result of the interactions with their peers during the simulations. These small groups of only 10 students were used to facilitate hands-on learning. This engages the students and faculty in discussion, which can enhance understanding of content. While the students engaged in peer learning, they also functioned as a team. In the current competencies, ${ }^{24}$ a team approach to practice is one of the foundational behaviors of professional practice. It is important to develop the students' ability to collaborate with fellow athletic trainers as well as other healthcare providers. ${ }^{25}$

\section{Organization of Thoughts}

Specific to the individual CBS, the organization of thoughts emerged as a theme. Participants perceived that the individual CBS helped them control and organize their thoughts during the evaluation as they performed the evaluation independently. Since these students are still novice in their evaluation skills, expert reasoning, such as case-pattern recognition, ${ }^{26}$ was likely not used when arriving at clinical decisions regarding the patient. Instead, the students were able to organize their thoughts during the evaluation. Geisler and Lazenby ${ }^{27}$ stated that it is important for students to learn to organize their knowledge into patterns and to be immersed in experiences that teach them how to think. Our participants had to draw from prior knowledge learned in their classes and/or clinical education. We believe the students may have been also refining their script during the CBS. Script acquisition and development of a script are extremely important at the beginning of a medical career. ${ }^{27}$

Scripts are described as attributes (eg, location of pain, degree of swelling, duration or frequency of pain) associated with values (eg, signs and symptoms) that have the probability of occurring. ${ }^{28}$ For example, a patient complains of anterior knee pain just above the tibial tuberosity with no mechanism of injury. A script for patellar tendonitis has attributes of location of pain, swelling, and type of pain, which have values assigned, such as no swelling, crepitus, and dull pain. From 
this information, the student immediately considers patellar tendonitis and proceeds with an evaluation to test this hypothesis. When beginning the evaluation, any value could fit (eg, bursitis), but the default value of patellar tendon pain is assumed until proven otherwise. When a patient presents, depending on the complaint, a script or network of information from previous knowledge and experiences is drawn upon to direct the questions and method of evaluation. Our participants were learning to organize their thoughts during the individual CBS experience. One participant commented about a deeper "understanding" of the evaluation process. As students repeatedly perform evaluations, they are exposed to new experiences and begin to reorganize their knowledge as efficiently as possible. ${ }^{28}$ Weaker or more inexperienced students operate from a disjointed list of signs and symptoms. The difference between "good" and "not so good" diagnostic thinking is not solely based on the amount of knowledge a person has but rather on how that knowledge is stored, organized, and used. ${ }^{29}$ Other healthcare professions have reported on scripts as a method to help develop students' clinical decision making to help students become more efficient in solving patient problems. ${ }^{28}$ Additional research is needed to determine when students begin script development and what specific educational interventions or clinical experiences can develop and redefine the script.

\section{Limitations and Further Research}

The individual CBS and small SP group encounters occurred at one university during a single semester, and the results may not represent the entire population of athletic training students. Additional research with a larger population over a longer period of time is needed. In addition, the participants reported they did not perform many evaluations on live patients during the course of the investigation. This limited the participants' ability to compare their learning experiences during the study to their evaluations of actual patients. Longitudinal studies conducted over longer periods of time (eg, months, years) are needed to determine how simulations and encounters with SPs can help educate and evaluate athletic training students. Lastly, the results represent the participants' perceived benefits of small SP group encounters and individual CBSs, and other perceived benefits could be present with more or different students.

The results from this investigation add to the literature in that simulations and SPs provide meaningful and realistic patient encounters for athletic training students. While we did find that these experiences increased student confidence and reflection-on-specific action, additional research is needed to determine how to best develop students' confidence and reflection-on-specific action during their educational preparation. Further investigations could include how factors such as time, group size, number of patient encounters, and feedback and interactions with preceptors affect confidence and reflection-on-specific action. Both of the experiences used in this investigation provided an opportunity for students to integrate knowledge and clinical skills in a realistic, nonthreatening environment with immediate feedback. During the individual CBS students began to develop, control, and organize their thoughts during the evaluation. Further research is needed to determine how students begin to organize their thoughts as well as develop scripts. Do scripts develop more efficiently when a student is providing patient care alone or in a group? In addition, does the level of student (ie, first year, second year) affect script development? Developing the cases for simulations/SPs, training the SP, and being present during the encounters is costly and time consuming for faculty. Research is needed to determine if group size in simulations and SP encounters has an effect on script development as well as other educational outcomes (eg, confidence, reflection-on-action, clinical decision making). Grouping students would allow for efficient teaching and evaluation. Our research shows students perceive learning from their peers when interacting in groups of 3 to 4 with a standardized patient. Interacting with peers as a part of a healthcare team when performing or planning patient care is an important skill to develop, and more research is needed to develop collaborative interaction.

A recent 10-year review ${ }^{9}$ of the literature on the use of SPs in teaching and learning recommended additional research measuring teaching interventions that result in clinicians and/or students changing their patient care based on their learning during an SP encounter. This behavior change could lead to better patient outcomes. ${ }^{9}$ To accomplish this, the use of rigorous research designs, which are either multicentered or use randomized control or comparison groups, was recommended. ${ }^{9}$ This investigation compared 2 teaching methods and found similarities and unique differences between each. Athletic training education would greatly benefit from additional research of current teaching methods to determine efficient and effective methods of utilizing simulations and SPs. This research could lead to best-practice teaching models in athletic training as well as improved patient outcomes.

\section{CONCLUSIONS}

Athletic training students need adequate clinical experiences that provide real-time opportunities to engage in patient care to develop them as future clinicians. Standardized patients and simulations are both being used to help students develop their athletic training knowledge and skills. ${ }^{2}$ Overall, the participants felt that both the individual CBS and the small SP group encounters increased their confidence and challenged them to reflect and think about future evaluations. Both the individual CBS and small SP group encounters provided realtime patient encounters in a nonthreatening manner. This provides the student an opportunity to learn in a safe environment without harming any patients. The participants engaged in peer learning and began to organize their thoughts. Simulations and SP encounters have the opportunity to engage students in patient interactions they may not normally see (eg, drug abuse, suicide) during clinical experiences, thus serving as an important and efficient educational tool. In addition, these experiences can be timed so they are concurrent with the content students are learning in the classroom and the laboratory.

\section{REFERENCES}

1. Walker SE, Weidner TG. Standardized patients provide realistic and worthwhile experiences for athletic training students. Athl Train Educ J. 2010;5(2):77-86.

2. Walker SE, Weidner TG, Armstrong KJ. Evaluation of athletic training students' clinical proficiencies. J Athl Train. 2008;43(4): 386-395. 
3. Armstrong KJ, Weidner TG, Walker SE. Athletic training approved clinical instructors report that more real-time opportunities are needed for evaluating clinical proficiencies. $J$ Athl Train. 2009;44(6):630-638.

4. Ebbert DW, Connors H. Standardized patient experiences: evaluation of clinical performance and nurse practitioner student satisfaction. Nurse Educ Perspect. 2004;25(1):12-15.

5. Yoo MS, Yoo IY. The effectiveness of standardized patients as a teaching method for nursing fundamentals. J Nurs Educ. 2003; 42(10):444-448.

6. Black B, Marcoux BC. Feasibility of using standardized patients in a physical therapist education program: a pilot study. $J$ Phys Ther Educ. 2002;16(2):49-56.

7. Jensen GM, Richert AE. Reflection on the teaching of ethics in physical therapist education: integrating cases, theory, and learning. J Phys Ther Educ. 2005;19(2):78-85.

8. Blatt B, Plack M, Maring J, Mintz M, Simmens SJ. Acting on reflection: the effect of reflection on students' clinical performance on a standardized patient examination. J Gen Intern Med. 2007;22(1):49-54.

9. May W, Park JH, Lee JP. A ten-year review of the literature on the use of standardized patients in teaching and learning: 19962005. Med Teach. 2009;31(6):487-492.

10. Barrows H. An overview of the uses of standardized patients for teaching and evaluating clinical skills. Acad Med. 1993;68(6): 443-445.

11. Strauss A, Corbin J. Basics of Qualitative Research: Techniques and Procedures for Developing Grounded Theory. 2nd ed. Thousand Oaks, CA: Sage; 1998.

12. Wainwright SF, Shepard KF, Harman LB, Stephens J. Novice and experienced physical therapist clinicians: a comparison of how reflection is used to inform the clinical decision-making process. Phys Ther. 2010;90(1):75-88.

13. Knight KL. Supervision of clinical education: a call for a paradigm shift. Athl Train Educ J. 2009;4(1):2-3.

14. Scriber K, Trowbridge C. Is direct supervision in clinical education for athletic training students always necessary to enhance student learning? Athl Train Educ J. 2009;4(1):32-37.

15. Dewey J. Democracy and Education. New York, NY: Free Press; 1944.
16. Leaver-Dunn D, Harrelson GL, Martin M, Wyatt T. Critical thinking pre-disposition among undergraduate athletic training students. J Athl Train. 2002;37(suppl 4):S147-S151.

17. Walker SE. Journaling writing as a teaching technique to promote reflection. J Athl Train. 2006;41(2):216-221.

18. Wainwright SF, Shepard KF, Harman LB, Stephens J. Factors that influence the clinical decision making of novice and experienced physical therapists. Phys Ther. 2011;91(1):87-101.

19. Henning JM, Weidner TG, Jones J. Peer-assisted learning in the athletic training clinical setting. J Athl Train. 2006;41(1):102108.

20. Euliano TY. Small group teaching: clinical correlations with a human patient simulator. Adv Physiol Educ. 2001;25(1-4):3643.

21. Weidner TG, Popp JK. Peer-assisted learning and orthopaedic evaluation psychomotor skills. J Athl Train. 2007;42(1):113-119.

22. Carr WD, Volberding J, Vardiman P. A peer-assisted learning program and its effect on student skill demonstration. Athl Train Educ J. 2011;6(3):129-135.

23. Ranse K, Grealish L. Nursing students' perceptions of learning in the clinical setting of the dedicated education unit. $J$ Adv Nurs. 2007;58(2):171-179.

24. National Athletic Trainers' Association. Athletic Training Education Competencies. 5th ed. Dallas, TX: National Athletic Trainers' Association; 2011.

25. Thistlethwaite J. Interprofessional education: a review of context, learning and the research agenda. Med Educ. 2012; 46(1):58-70.

26. Charlin B, Boshuizen HP, Custers EJ, Feltovich PJ. Scripts and clinical reasoning. Med Educ. 2007;41(12):1178-1184.

27. Geisler PR, Lazenby TW. Clinical reasoning in athletic training education: modeling expert thinking. Athl Train Educ J. 2009; $4(2): 52-65$.

28. Charlin B, Tardif J, Boshuizen H. Scripts and medical diagnostic knowledge: theory and applications for clinical reasoning instruction and research. Acad Med. 2000;75(2):182-190.

29. Bordage G, Lemieux M. Semantic structures and diagnostic thinking of experts and novices. Acad Med. 1991;66(suppl 9): S70-S72. 